Appl Math Optim 46:81-88 (2002)

DOI: $10.1007 / \mathrm{s} 00245-002-0754-2$

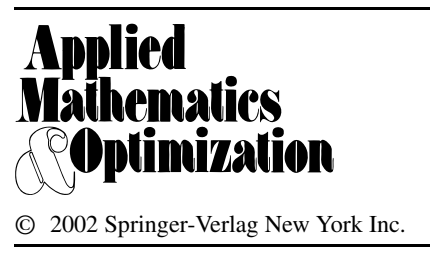

\title{
Nonlinear Filtering with Fractional Brownian Motion
}

\author{
Anna Amirdjanova \\ Department of Statistics, University of Michigan, \\ Ann Arbor, MI 48109-1092, USA
}

\begin{abstract}
Our objective is to study a nonlinear filtering problem for the observation process perturbed by a Fractional Brownian Motion (FBM) with Hurst index $\frac{1}{2}<H<1$. A reproducing kernel Hilbert space for the FBM is considered and a "fractional" Zakai equation for the unnormalized optimal filter is derived.
\end{abstract}

Key Words. Nonlinear filtering, Fractional Brownian motion, Reproducing kernel Hilbert space, Stochastic differential equations.

AMS Classification. 60H20, 60G15, 60G35.

\section{Introduction}

The goal of nonlinear filtering theory is to estimate a signal process $\left(X_{t}\right)(0 \leq t \leq T)$ observed in the presence of an additive noise. Consider a complete probability space $(\Omega, \mathcal{F}, P)$ and a family $\left(\mathcal{F}_{t}\right)_{t \geq 0}$ of right-continuous increasing $P$-complete sub- $\sigma$-fields of $\mathcal{F}$. Let $X=\left(X_{t}, t \in[0, T]\right)$ be a measurable, $\mathcal{F}_{t}$-adapted stochastic process with values in a complete separable metric space $S$. The simplest model for the observation process $\left(Y_{t}\right)$ is given by

$$
Y_{t}=\int_{0}^{t} h\left(X_{s}\right) d s+B_{t}, \quad 0 \leq t \leq T,
$$

where $\left(B_{t}\right)$ is a standard Brownian Motion (BM), and $h \in C(S)$ satisfies

$$
\int_{0}^{T} h^{2}\left(X_{s}\right)(\omega) d s<\infty \quad(P \text {-a.s. }) .
$$

The classical model (1)-(2) can be written in the following form:

$$
Y_{t}(\omega)=F_{t}(X(\omega))+B_{t}(\omega), \quad t \in[0, T],
$$


where $\left\{F_{t}(X(\omega)), t \in[0, T]\right\}$ is ( $P$-a.s.) an element of the Reproducing Kernel Hilbert Space (RKHS) of the BM on $[0, T]$.

The objective of this paper is to investigate a nonlinear filtering problem in the case when an additive observation noise exhibits a certain long-range dependence structure. Namely, let $\left(B_{t}^{H}, t \in[0, T]\right)$ be a Fractional Brownian Motion (FBM) with (fixed) Hurst index $\frac{1}{2}<H<1$ and let $\mathcal{H}\left(B^{H}\right)$ stand for the RKHS of $\left(B_{t}^{H}, t \in[0, T]\right)$. We study the following analogue of the model (3):

$$
Y_{t}(\omega)=F_{t}(X(\omega))+B_{t}^{H}(\omega), \quad t \in[0, T],
$$

where $\left\{F(X(\omega)\} \in \mathcal{H}\left(B^{H}\right)\right.$ for almost all $\omega$.

In Section 2 we present important properties of the RKHS $\mathcal{H}\left(B^{H}\right)$ and give a more explicit form to the observation model (4). Here we use the results on $\mathcal{H}\left(B^{H}\right)$ obtained by Barton and Poor [1]. In Section 3 we derive a Bayes' formula for the optimal filter for the observation model with FBM noise and state a corresponding "fractional" Zakai-type equation for the unnormalized conditional expectation.

Let us give a few comments on other types of filtering models considered in the literature. Interesting results on linear filtering with FBM were obtained by Kleptsyna et al. in [5]-[7] and Le Breton in [8]. As far as nonlinear theory is concerned, Coutin and Decreusefond in [2] considered a nonlinear filtering model where both the signal and the observation are solutions of a stochastic differential equation driven by a multidimensional FBM. A nonlinear filtering model with FBM in the signal process (and a Brownian component driving the observation process) was also investigated by Kleptsyna et al. in [4].

\section{RKHS $\mathcal{H}\left(B^{H}\right)$ and the Observation Process Model}

Let us fix a complete probability space $(\Omega, \mathcal{F}, P)$ on which all random processes are defined. For a given $H \in\left(\frac{1}{2}, 1\right)$, let $B^{H}=\left(B_{t}^{H}, t \in[0, T]\right)$ be an FBM with Hurst index $H$. Namely, $B^{H}$ has the following properties:

(i) $B^{H}$ is a Gaussian process with continuous sample paths and stationary increments.

(ii) $B_{0}^{H}=0, E B_{t}^{H}=0$ for all $t \geq 0$ and the covariance kernel is given by

$$
R_{H}\left(t_{1}, t_{2}\right):=E B_{t_{1}}^{H} B_{t_{2}}^{H}=\frac{c^{2}}{2}\left\{\left|t_{1}\right|^{2 H}+\left|t_{2}\right|^{2 H}-\left|t_{1}-t_{2}\right|^{2 H}\right\},
$$

where

$$
c^{2}:=\operatorname{Var}\left(B_{1}^{H}\right)=-\frac{\Gamma(2-2 H) \cos (\pi H)}{\pi H(2 H-1)} .
$$

It is well known that $B^{H}$ is self-similar with self-similarity index $H, B^{H}$ is not a semimartingale and (since $H \in\left(\frac{1}{2}, 1\right)$ ) has a long-range dependence structure, i.e.

$$
\sum_{n=0}^{\infty} \operatorname{Cov}\left(B_{1}^{H}, B_{n+1}^{H}-B_{n}^{H}\right)=\infty .
$$


Let $\mathcal{H}\left(B^{H}\right)$ denote the RKHS of $B^{H}=\left(B_{t}^{H}, t \in[0, T]\right)$. Then $\mathcal{H}\left(B^{H}\right)$ satisfies the following conditions:

(i) $\mathcal{H}\left(B^{H}\right)$ is a Hilbert space of real-valued functions on $[0, T]$.

(ii) $\forall t \in[0, T], R_{H}(\cdot, t) \equiv \operatorname{Cov}\left(B^{H}, B_{t}^{H}\right) \in \mathcal{H}\left(B^{H}\right)$.

(iii) $\forall g \in \mathcal{H}\left(B^{H}\right),\left\langle g(\cdot), R_{H}(\cdot, t)\right\rangle_{\mathcal{H}\left(B^{H}\right)}=g(t)$.

Specifically, by Theorem 4.4 in [1], for $\frac{1}{2}<H<1, \mathcal{H}\left(B^{H}\right)$ consists of functions of the form

$$
g(t)=\int_{0}^{t} g^{*}(s) \gamma_{H}(s, t) d s, \quad t \in[0, T],
$$

where $g^{*} \in L^{2}([0, T])$,

$$
\gamma_{H}(s, t):=\frac{s^{1 / 2-H}}{\Gamma\left(H-\frac{1}{2}\right)} \int_{s}^{t} \tau^{H-1 / 2}(\tau-s)^{H-3 / 2} d \tau,
$$

and $\forall g_{1}, g_{2} \in \mathcal{H}\left(B^{H}\right)$,

$$
\left\langle g_{1}, g_{2}\right\rangle_{\mathcal{H}\left(B^{H}\right)}=\int_{0}^{T} g_{1}^{*}(s) g_{2}^{*}(s) d s .
$$

Note that any $g$ from $\mathcal{H}\left(B^{H}\right)$ has a derivative almost everywhere in $[0, T]$. For almost all $t \in[0, T]$, the relationship (6) between $g$ and $g^{*}$ can be inverted:

$$
g^{*}(t)=t^{H-1 / 2} \frac{d}{d t}\left(\int_{0}^{t} k(t, \tau) \frac{\partial}{\partial \tau} g(\tau) d \tau\right),
$$

where

$$
k(t, \tau):=\frac{1}{\Gamma\left(\frac{3}{2}-H\right)}(t-\tau)^{1 / 2-H} \tau^{1 / 2-H} .
$$

The observation model (4) can then be written as

$$
Y_{t}(\omega)=\int_{0}^{t} h\left(X_{s}(\omega)\right) \gamma_{H}(s, t) d s+B_{t}^{H}(\omega), \quad t \in[0, T],
$$

where

$$
\gamma_{H}(s, t)=\frac{s^{1 / 2-H}}{\Gamma\left(H-\frac{1}{2}\right)} \int_{s}^{t} \tau^{H-1 / 2}(\tau-s)^{H-3 / 2} d \tau,
$$

and $h \in C(S)$ satisfies

$$
\int_{0}^{T} h^{2}\left(X_{s}\right)(\omega) d s<\infty \quad(P \text {-a.s. }) .
$$

Let us also assume the following condition:

$$
\left(X_{t}\right) \text { is independent of }\left(B_{t}^{H}\right) \text {. }
$$




\section{Bayes' Formula and a "Fractional" Zakai-Type Equation}

Let $\left(\tilde{\mathcal{F}}_{t}^{X}\right)$ be the natural family of $X$, and let $\left(\tilde{\mathcal{F}}_{t}^{B^{H}}\right)$ be the natural family of $B^{H}$. Since $X$ is independent of $B^{H}$, we can assume that $\left(X_{t}\right)$ is defined on $\left(\tilde{\Omega}^{X}, \tilde{\mathcal{F}}^{X},\left(\tilde{\mathcal{F}}_{t}^{X}\right), \tilde{P}^{X}\right)$, and $\left(B_{t}^{H}\right)$ is defined on $\left(\tilde{\Omega}^{B^{H}}, \tilde{\mathcal{F}}^{B^{H}},\left(\tilde{\mathcal{F}}_{t}^{B^{H}}\right), \tilde{P}^{B^{H}}\right)$, where $\Omega=\tilde{\Omega}^{X} \times \tilde{\Omega}^{B^{H}}, \mathcal{F}=\tilde{\mathcal{F}}^{X} \times \tilde{\mathcal{F}}^{B^{H}}$, $P=\tilde{P}^{X} \times \tilde{P}^{B^{H}}$. Define $\mathcal{F}_{t}^{X}=\tilde{\mathcal{F}}_{t}^{X} \times\left\{\emptyset, \tilde{\Omega}^{B^{H}}\right\}, \mathcal{F}_{t}^{B^{H}}=\left\{\emptyset, \tilde{\Omega}^{X}\right\} \times \tilde{\mathcal{F}}_{t}^{B^{H}}$ and put $\mathcal{F}_{t}^{Y}=\sigma\left\{Y_{s}, 0 \leq s \leq t\right\}$. Let $P^{Y}$ be the restriction of $P$ to $\mathcal{F}_{T}^{Y}$.

Theorem 1. Assume the conditions of the model (11)-(13). Then for any integrable and $\tilde{\mathcal{F}}_{T}^{X}$-measurable function $f$, we have that $\left(P^{Y}\right.$-a.s. $)$

$$
E\left[f \mid \mathcal{F}_{t}^{Y}\right](\omega)=\frac{\int_{\tilde{\Omega}^{X}} f\left(u^{\prime}\right) \exp \left\{\alpha_{u^{\prime}}(t)(\omega)\right\} \tilde{P}^{X}\left(d u^{\prime}\right)}{\int_{\tilde{\Omega}^{X}} \exp \left\{\alpha_{u^{\prime}}(t)(\omega)\right\} \tilde{P}^{X}\left(d u^{\prime}\right)},
$$

where

$$
\begin{aligned}
\alpha_{u^{\prime}}(t)(\omega)= & \int_{0}^{t} h\left(X_{s}\left(u^{\prime}\right)\right) s^{H-1 / 2} d\left(\int_{0}^{s} k(s, \tau) d Y_{\tau}(\omega)\right) \\
& -\frac{1}{2} \int_{0}^{t}\left[h\left(X_{s}\left(u^{\prime}\right)\right)\right]^{2} d s,
\end{aligned}
$$

and

$$
k(s, \tau)=\frac{1}{\Gamma\left(\frac{3}{2}-H\right)}(s-\tau)^{1 / 2-H} \tau^{1 / 2-H} .
$$

Proof. The proof is based on the reference probability method. Let $Q(\cdot, \omega)$ be a version of the conditional probability relative to $\mathcal{F}_{T}^{X}$ on the $\sigma$-field $\mathcal{F}_{t}^{Y}$, i.e.

$$
Q(A, \omega)=E\left(1_{A} \mid \mathcal{F}_{T}^{X}\right)(\omega) \quad(P \text {-a.s. }),
$$

$\forall A \in \mathcal{F}_{t}^{Y}$. Then, $\forall \omega^{\prime}=\left(u^{\prime}, v^{\prime}\right) \in \Omega, A \in \mathcal{F}_{t}^{Y}$,

$$
Q\left(A, \omega^{\prime}\right)=\delta_{u^{\prime}} \times \tilde{P}^{B^{H}}(A),
$$

where $\delta_{u^{\prime}}$ is a probability measure on $\tilde{\mathcal{F}}_{T}^{X}$ with total mass concentrated at $\left\{u^{\prime}\right\}$.

Moreover, under the law $\delta_{u^{\prime}} \times \tilde{P}^{B^{H}},\left(B_{t}^{H}\right)$ is an FBM and

$$
\left.Y_{t}(\omega)=\int_{0}^{t} \gamma_{H}(s, t) h\left(X_{s}\left(u^{\prime}\right)\right) d s+B_{t}^{H}(\omega) \quad \text { (a.s. }\right),
$$

$\forall t \in[0, T]$. Define $\hat{\mathcal{F}}_{t}^{Y}=\mathcal{F}_{t}^{Y} \vee\left\{\right.$ all $Q\left(\cdot, \omega^{\prime}\right)$-null sets in $\left.\mathcal{F}_{T}^{Y}\right\}$. Then $B_{t}^{H}(\omega)$ and $Y_{t}(\omega)$ are both $\hat{\mathcal{F}}_{t}^{Y}$-adapted and, under $Q\left(\cdot, \omega^{\prime}\right)$, are FBMs with mean functions zero and $\beta\left(t ; u^{\prime}\right)$, respectively, where

$$
\beta\left(t ; u^{\prime}\right)=\int_{0}^{t} \gamma_{H}(s, t) h\left(X_{s}\left(u^{\prime}\right)\right) d s, \quad t \in[0, T] .
$$


Let

$$
\tilde{\Omega}_{0}^{X}:=\left\{u \in \tilde{\Omega}^{X}: \int_{0}^{T} h^{2}\left(X_{s}(u)\right) d s<\infty\right\}
$$

then $\tilde{P}^{X}\left(\tilde{\Omega}_{0}^{X}\right)=1$ and $\forall u \in \tilde{\Omega}_{0}^{X}, \beta(\cdot ; u) \in \mathcal{H}\left(B^{H}\right)$. Let us denote by $\mathcal{H}\left(B^{H \mid t}\right)$ the RKHS of $B^{H}$ restricted to $[0, t]$. Then, $\forall u \in \tilde{\Omega}_{0}^{X}, \beta(\cdot ; u)$ viewed as a function on $[0, t]$ belongs to $\mathcal{H}\left(B^{H \mid t}\right)$. Let $\Omega_{0}=\tilde{\Omega}_{0}^{X} \times \tilde{\Omega}^{B^{H}}$. Fix $\omega^{\prime}=\left(u^{\prime}, v^{\prime}\right) \in \Omega_{0}$. Since $Q \circ\left(B^{H}\right)^{-1}\left(\cdot, \omega^{\prime}\right) \equiv$ $Q\left(\cdot, \omega^{\prime}\right) \circ\left(B^{H}\right)^{-1}$ and $Q \circ Y^{-1}\left(\cdot, \omega^{\prime}\right) \equiv Q\left(\cdot, \omega^{\prime}\right) \circ Y^{-1}$ are Gaussian measures on $C[0, t]$ with the common covariance kernel

$$
R_{H}\left(s_{1}, s_{2}\right)=\frac{c^{2}}{2}\left\{\left|s_{1}\right|^{2 H}+\left|s_{2}\right|^{2 H}-\left|s_{1}-s_{2}\right|^{2 H}\right\}
$$

and mean functions 0 and $\left(\beta\left(s ; u^{\prime}\right), 0 \leq s \leq t\right)$ from $\mathcal{H}\left(B^{H \mid t}\right)$, by Theorem 5A in [9], the two measures are mutually absolutely continuous and the Radon-Nikodym derivative is given by

$$
\frac{d Q \circ Y^{-1}}{d Q \circ\left(B^{H}\right)^{-1}}(Y)=\exp \left\{\left\langle Y, \beta\left(\cdot ; u^{\prime}\right)\right\rangle_{t}-\frac{1}{2}\left\|\beta\left(\cdot ; u^{\prime}\right)\right\|_{\mathcal{H}\left(B^{H||^{\prime}}\right)}^{2}\right\},
$$

where

$$
\left\langle Y, \beta\left(\cdot ; u^{\prime}\right)\right\rangle_{t} \equiv\left\langle\beta\left(\cdot ; u^{\prime}\right)+B^{H \mid t}, \beta\left(\cdot ; u^{\prime}\right)\right\rangle_{t}:=\left\|\beta\left(\cdot ; u^{\prime}\right)\right\|_{\mathcal{H}\left(B^{H \mid t}\right)}^{2}+\varphi\left(\beta\left(\cdot ; u^{\prime}\right)\right),
$$

and $\varphi$ is the congruence satisfying the following conditions:

(i) $\varphi: \mathcal{H}\left(B^{H \mid t}\right) \rightarrow L^{2}\left(B^{H \mid t}\right)$.

(ii) $\varphi\left(R_{H}(\cdot, s)\right)=B_{s}^{H}, \forall s \in[0, t]$.

(iii) $E[\varphi(g)]=0, \operatorname{Cov}\left[\varphi\left(g_{1}\right), \varphi\left(g_{2}\right)\right]=\left\langle g_{1}, g_{2}\right\rangle_{\mathcal{H}\left(B^{H \mid t}\right)}$.

Consider an orthogonal increment process $\left(Z_{t}\right)$ given by

$$
Z_{t}=\int_{0}^{t} k(t, \tau) d B_{\tau}^{H}, \quad t \in[0, T],
$$

with the kernel $k(t, \tau)$ defined by (10). Then one can show (see [1]) that

$$
\left\langle B^{H \mid t}, g\right\rangle_{t}:=\varphi(g)=\int_{0}^{t} g^{*}(s) s^{H-1 / 2} d Z_{s}, \quad \forall g \in \mathcal{H}\left(B^{H \mid t}\right) .
$$

For $\beta\left(\cdot ; u^{\prime}\right) \in \mathcal{H}\left(B^{H \mid t}\right)$, the function $h\left(X .\left(u^{\prime}\right)\right)$ plays the role of $\beta^{*}\left(\cdot ; u^{\prime}\right)$. Thus,

$$
\varphi\left(\beta\left(\cdot ; u^{\prime}\right)\right)=\int_{0}^{t} h\left(X_{s}\left(u^{\prime}\right)\right) s^{H-1 / 2} d Z_{s}
$$

and

$$
\left\|\beta\left(\cdot ; u^{\prime}\right)\right\|_{\mathcal{H}\left(B^{H \mid t}\right)}^{2}=\int_{0}^{t}\left[h\left(X_{s}\left(u^{\prime}\right)\right)\right]^{2} d s .
$$


Let

$$
\alpha_{u^{\prime}}(t)(\omega):=\left\langle Y(\omega), \beta\left(\cdot, u^{\prime}\right)\right\rangle_{t}-\frac{1}{2}\left\|\beta\left(\cdot, u^{\prime}\right)\right\|_{\mathcal{H}\left(B^{H \mid t}\right)}^{2} .
$$

Then

$$
\alpha_{u^{\prime}}(t)=\int_{0}^{t} h\left(X_{s}\left(u^{\prime}\right)\right) s^{H-1 / 2} d Z_{s}+\frac{1}{2} \int_{0}^{t}\left[h\left(X_{s}\left(u^{\prime}\right)\right)\right]^{2} d s,
$$

where

$$
Z_{t}=\int_{0}^{t} k(t, s) d B_{s}^{H}=\int_{0}^{t} k(t, s) d Y_{s}-\int_{0}^{t} k(t, s) \frac{\partial}{\partial s} \beta\left(s, u^{\prime}\right) d s,
$$

and, in view of (9),

$$
Z_{t}=\int_{0}^{t} k(t, s) d Y_{s}-\int_{0}^{t} s^{1 / 2-H} h\left(X_{s}\left(u^{\prime}\right)\right) d s .
$$

Therefore, we obtain that (24) has the following form:

$$
\begin{aligned}
\alpha_{u^{\prime}}(t)(\omega)= & \int_{0}^{t} h\left(X_{s}\left(u^{\prime}\right)\right) s^{H-1 / 2} d\left(\int_{0}^{s} k(s, \tau) d Y_{\tau}(\omega)\right) \\
& -\frac{1}{2} \int_{0}^{t}\left[h\left(X_{s}\left(u^{\prime}\right)\right)\right]^{2} d s .
\end{aligned}
$$

Consider the measure $\lambda_{\omega^{\prime}}$ on $\mathcal{F}_{t}^{Y}$ given by

$$
d \lambda_{\omega^{\prime}}(\cdot)=\exp \left\{-\alpha_{u^{\prime}}(t)\right\} d Q\left(\cdot, \omega^{\prime}\right) .
$$

Under $Q\left(\cdot, \omega^{\prime}\right)$,

$$
\int_{0}^{t} h\left(X_{s}\left(u^{\prime}\right)\right) s^{H-\frac{1}{2}} d Z_{s} \sim \mathcal{N}\left(0, \int_{0}^{t}\left[h\left(X_{s}\left(u^{\prime}\right)\right)\right]^{2} d s\right) .
$$

Then $\lambda_{\omega^{\prime}}$ is a probability measure on $\mathcal{F}_{t}^{Y}$ and $Y$ is a mean-zero FBM under $\lambda_{\omega^{\prime}}$. Also $\lambda_{\omega_{1}^{\prime}}=\lambda_{\omega_{2}^{\prime}}$ on $\mathcal{F}_{t}^{Y}$, i.e. it does not depend on $\omega^{\prime}$. Let us call it just $\lambda$. By Lemma 11.3.3 in [3] ( $\lambda$-a.s. $)$,

$$
\exists q\left(\omega, \omega^{\prime}\right)=\frac{d Q\left(\cdot, \omega^{\prime}\right)}{d \lambda}(\omega)=\exp \left\{\alpha_{u^{\prime}}(t)(\omega)\right\},
$$

which is $\left(\mathcal{F}_{t}^{Y} \times \mathcal{F}_{T}^{X}\right)$-measurable, and for $\tilde{\mathcal{F}}_{T}^{X}$-measurable and integrable function $f$,

$$
E\left[f \mid \mathcal{F}_{t}^{Y}\right](\omega)=\frac{\int_{\tilde{\Omega}^{X}} f\left(u^{\prime}\right) \exp \left\{\alpha_{u^{\prime}}(t)(\omega)\right\} \tilde{P}^{X}\left(d u^{\prime}\right)}{\int_{\tilde{\Omega}^{X}} \exp \left\{\alpha_{u^{\prime}}(t)(\omega)\right\} \tilde{P}^{X}\left(d u^{\prime}\right)} \quad\left(P^{Y} \text {-a.s. }\right) .
$$


Remark 1. Bayes' formula for the model (11)-(13) could be obtained directly from the classical Bayes' formula with the help of the following integral representation of the FBM (Theorem 4.5 in [1]):

$$
\begin{aligned}
& \exists\left(B_{t}, 0 \leq t \leq T\right), \text { a standard BM, such that } \forall t \in[0, T], \\
& B_{t}^{H}(\omega)=\int_{0}^{t} \gamma_{H}(s, t) d B_{s}(\omega) \quad(P \text {-a.s. }) .
\end{aligned}
$$

Namely, let

$$
\tilde{Y}_{t}(\omega):=\int_{0}^{t} h\left(X_{s}(\omega)\right) d s+B_{t}(\omega) \quad(P \text {-a.s. })
$$

for $t \in[0, T]$. Then

$$
Y_{t}(\omega)=\int_{0}^{t} \gamma_{H}(s, t) d \tilde{Y}_{s}(\omega), \quad 0 \leq t \leq T
$$

We can invert the above relationship between $Y$ and $\tilde{Y}$ ( $P$-a.s.),

$$
\tilde{Y}_{t}=\int_{0}^{t} s^{H-1 / 2} d\left(\int_{0}^{s} k(s, \tau) d Y_{\tau}\right)
$$

and note that $\mathcal{F}_{t}^{Y}=\mathcal{F}_{t}^{\tilde{Y}}$ for all $t$. Thus, $E\left[f \mid \mathcal{F}_{t}^{Y}\right](\omega)=E\left[f \mid \mathcal{F}_{t}^{\tilde{Y}}\right]$ almost surely, and using a Bayes' formula for the classical model (28) and equation (29), one obtains the desired result.

Theorem 2. Assume that $\left(X_{t}\right)$ is an $S$-valued Markov process with the generator $L$ with domain $\mathcal{D}$. Moreover, assume that the paths of $\left(X_{t}\right)$ are progressively measurable, and $E_{P} \int_{0}^{T}\left|f\left(X_{s}\right)\right|^{2} d s<\infty$ for all $f \in \mathcal{D}_{0}$, where $\mathcal{D}_{0}$ consists of all $f: S \rightarrow \mathbb{R}$ such that $f_{1}$ defined by $f_{1}(s, x):=f(x)$ belong to $\mathcal{D}$. For $f \in \mathcal{D}_{0}$ let us put $\left(L_{t} f\right)(x):=\left(L f_{1}\right)(t, x)$. For the observation model (11)-(13) define

$$
\sigma_{t}(f, Y)(\omega)=\int_{\tilde{\Omega}^{X}} f\left(u^{\prime}\right) \exp \left\{\alpha_{u^{\prime}}(t)(\omega)\right\} \tilde{P}^{X}\left(d u^{\prime}\right),
$$

where

$$
\begin{aligned}
\alpha_{u^{\prime}}(t)(\omega)= & \int_{0}^{t} h\left(X_{s}\left(u^{\prime}\right)\right) s^{H-1 / 2} d\left(\int_{0}^{s} k(s, \tau) d Y_{\tau}(\omega)\right) \\
& -\frac{1}{2} \int_{0}^{t}\left[h\left(X_{s}\left(u^{\prime}\right)\right)\right]^{2} d s .
\end{aligned}
$$

Then for all $f \in \mathcal{D}_{0}, \sigma_{t}(f, Y)$ satisfies the following Zakai-type equation:

$$
d \sigma_{t}(f, Y)=\sigma_{t}\left(L_{t} f, Y\right) d t+\sigma_{t}(h f, Y) t^{H-1 / 2} d\left[\int_{0}^{t} k(t, s) d Y_{s}\right]
$$

with

$$
k(t, s)=\frac{1}{\Gamma\left(\frac{3}{2}-H\right)}(t-s)^{1 / 2-H} s^{1 / 2-H} .
$$


Proof. The equation follows immediately from the Zakai equation for the classical observation model (28) and Remark 1.

\section{References}

1. R.J. Barton, H.V. Poor, Signal detection in fractional Gaussian noise, IEEE Trans. Inform. Theory 34(5) (1988), 943-959.

2. L. Coutin, L. Decreusefond, Abstract nonlinear filtering theory in the presence of fractional Brownian motion, Ann. Appl. Probab. 9(4) (1999), 1058-1090.

3. G. Kallianpur, Stochastic Filtering Theory, Springer-Verlag, New York, 1980.

4. M.L. Kleptsyna, P.E. Kloeden, V.V. Anh, Nonlinear filtering for processes with fractional Brownian motion (in Russian), Problemy Peredachi Informatsii 34(2) (1998), 65-76.

5. M.L. Kleptsyna, P.E. Kloeden, V.V. Anh, Linear filtering with fractional Brownian motion, Stochastic Anal. Appl. 16(5) (1998), 907-914.

6. M.L. Kleptsyna, P.E. Kloeden, V.V. Anh, Linear filtering with fractional Brownian motion in the signal and observation processes, J. Appl. Math. Stochastic Anal. 12(1) (1999), 85-90.

7. M.L. Kleptsyna, A. Le Breton, M.-C. Roubaud, General approach to filtering with fractional Brownian noises-applications to linear systems, Stochastics Stochastics Rep. 71(1-2) (2000), 119-140.

8. A. Le Breton, Filtering and parameter estimation in a simple linear system driven by a fractional Brownian motion, Statistics Probab. Lett. 38 (1998), 263-274.

9. E. Parzen, Regression analysis of continuous parameter time series, Proc. 4th Berkeley Sympos. Math. Statist. Probab., vol. I, pp. 469-489, University of California Press, Berkeley, CA, 1961.

Online publication 25 November 2002. 